\title{
Combinatorial engineering of hybrid mevalonate pathways in Escherichia coli for protoilludene production
}

\author{
Liyang Yang ${ }^{1 \dagger}$, Chonglong Wang ${ }^{1 \dagger}$, Jia Zhou ${ }^{1,2}$ and Seon-Won Kim ${ }^{1 *}$
}

\begin{abstract}
Background: Protoilludene is a valuable sesquiterpene and serves as a precursor for several medicinal compounds and antimicrobial chemicals. It can be synthesized by heterologous expression of protoilludene synthase in Escherichia coli with overexpression of mevalonate (MVA) or methylerythritol-phosphate (MEP) pathway, and farnesyl diphosphate (FPP) synthase. Here, we present E. coli as a cell factory for protoilludene production.

Results: Protoilludene was successfully produced in E. coli by overexpression of a hybrid exogenous MVA pathway, endogenous FPP synthase (IspA), and protoilludene synthase (OMP7) of Omphalotus olearius. For improving protoilludene production, the MVA pathway was engineered to increase synthesis of building blocks isopentenyl diphosphate (IPP) and dimethylallyl diphosphate (DMAPP) by sequential order permutation of the lower MVA portion (MVL), the alteration of promoters and copy numbers for the upper MVA portion (MvU), and the coordination of both portions, resulting in an efficient entire MVA pathway. To reduce the accumulation of mevalonate observed in the culture broth due to lower efficiency of the MvL than the MvU, the MvL was further engineered by homolog substitution with the corresponding genes from Staphylococcus aureus. Finally, the highest protoilludene production of $1199 \mathrm{mg} / \mathrm{L}$ was obtained from recombinant E. coli harboring the optimized hybrid MVA pathway in a test tube culture.
\end{abstract}

Conclusions: This is the first report of microbial synthesis of protoilludene by using an engineered $E$. coli strain. The protoilludene production was increased by approx. Thousandfold from an initial titer of $1.14 \mathrm{mg} / \mathrm{L}$. The strategies of both the sequential order permutation and homolog substitution could provide a new perspective of engineering MVA pathway, and be applied to optimization of other metabolic pathways.

Keywords: Protoilludene, Escherichia coli, Mevalonate pathway, Sequential order permutation, Homolog substitution

\section{Background}

Protoilludene derivatives, including illudins, marasmanes and melleolides, are known to exert antitumor and antimicrobial activities [1-3]. For example, the most brilliant potential anticancer agent illudin $\mathrm{S}$, which is first isolated from Omphalotus olearius mushroom, has been studied extensively owing to its cytotoxicity to various tumor cell types [4]. These biological properties and medicinal potential have attracted considerable attention since the

\footnotetext{
*Correspondence: swkim@gnu.ac.kr

'Liyang Yang and Chonglong Wang contributed equally to this work

1 Division of Applied Life Science (BK21 Plus Program), PMBBRC,

Gyeongsang National University, Jinju 660-701, Korea

Full list of author information is available at the end of the article
}

late 1960s. Illudins, marasmanes and melleolides can be synthesized from protoilludene by different oxygenation reactions. For example, P450 monooxygenases for the biosynthesis of illudin have been identified from $O$. olearius [5]. However, protoilludene is naturally produced in a small quantity and its purification from biological material suffers from low yields. Hence, metabolic engineering of microorganisms, such as Escherichia coli, is an alternative and attractive route for the production of protoilludene.

Protoilludene biosynthesis begins with the formation of the universal precursors, isopentenyl pyrophosphate (IPP) and dimethylallyl pyrophosphate (DMAPP), which can be generated 
via the methylerythritol-phosphate (MEP) pathway and the mevalonate (MVA) pathway [6]. Isopentenyl pyrophosphate and dimethylallyl pyrophosphate are condensed to form farnesyl diphosphate (FPP) by FPP synthase. Linear FPP undergoes multiple electrophilic cyclizations and rearrangements to generate tricyclic protoilludene with an action of protoilludene synthase, which has been isolated from various species including O. olearius, Armillaria gallica, and Stereum hirsutum [7-9]. O. olearius protoilludene synthase (OMP7) exhibits a superior catalytic efficiency $\left(\mathrm{K}_{\text {cat }} / \mathrm{K}_{\mathrm{m}}\right)$ of $(13.0 \pm 2.0) \times 10^{4} \mathrm{M}^{-1} \mathrm{~s}^{-1}$ among those protoilludene synthases (Additional file 1: Table S1) [8].

The entire protoilludene synthesis pathway via the MVA pathway can be divided into three portions, referred to as "MvU" composed of acetyl-CoA acetyltransferase/3-hydroxy-3-methylglutaryl-CoA reductase (MvaE) and 3-hydroxy-3-methylglutarylCoA synthase (MvaS), "MvL" composed of mevalonate kinase (MvaK1), phosphomevalonate kinase (MvaK2), diphosphomevalonate decarboxylase (MvaD) and IPP isomerase (IDI), and "AO" composed of FPP synthase (IspA) and protoilludene synthase (OMP7) (Fig. 1). The MVA pathway has been widely engineered for production of several sesquiterpenes in E. coli [6, 10-12]. In this study, MVA pathway was engineered for a balanced expression of $\mathrm{MvU}$ and $\mathrm{MvL}$ portions to increase protoilludene production. The MvL portion was optimized by sequential permutation of its constituent genes in consideration of transcriptional polarity, a general tendency of lower expression of the genes distant from promoter in a multi-cistronic operon [13]. In the optimized MvL portion by the random sequential permutation, the constituent genes would be arranged in their activities from low to high activities in the operon. The expression of $\mathrm{MvU}$ portion was modulated by changes of promoters and copy-numbers to tune mevalonate production to its utilization by MvL portion. Optimal coordination of the MvUs and MvLs portions of the MVA pathway were finally able to increase protoilludene production from 1.14 to $721 \mathrm{mg} / \mathrm{L}$. As accumulation of mevalonate intermediate was observed in the culture broth, MvL portion was further engineered by substituting its constituent genes with their homologues from Staphylococcus aureus. By the homolog substitution, protoilludene production was increased from 721 to $1199 \mathrm{mg} / \mathrm{L}$ in a test tube culture. The successful production of protoilludene from E. coli is shown in this work and the recombinant $E$. coli harboring the combinatorially engineered hybrid MVA pathway can serve as a basic platform host for production of other valuable terpenoids.

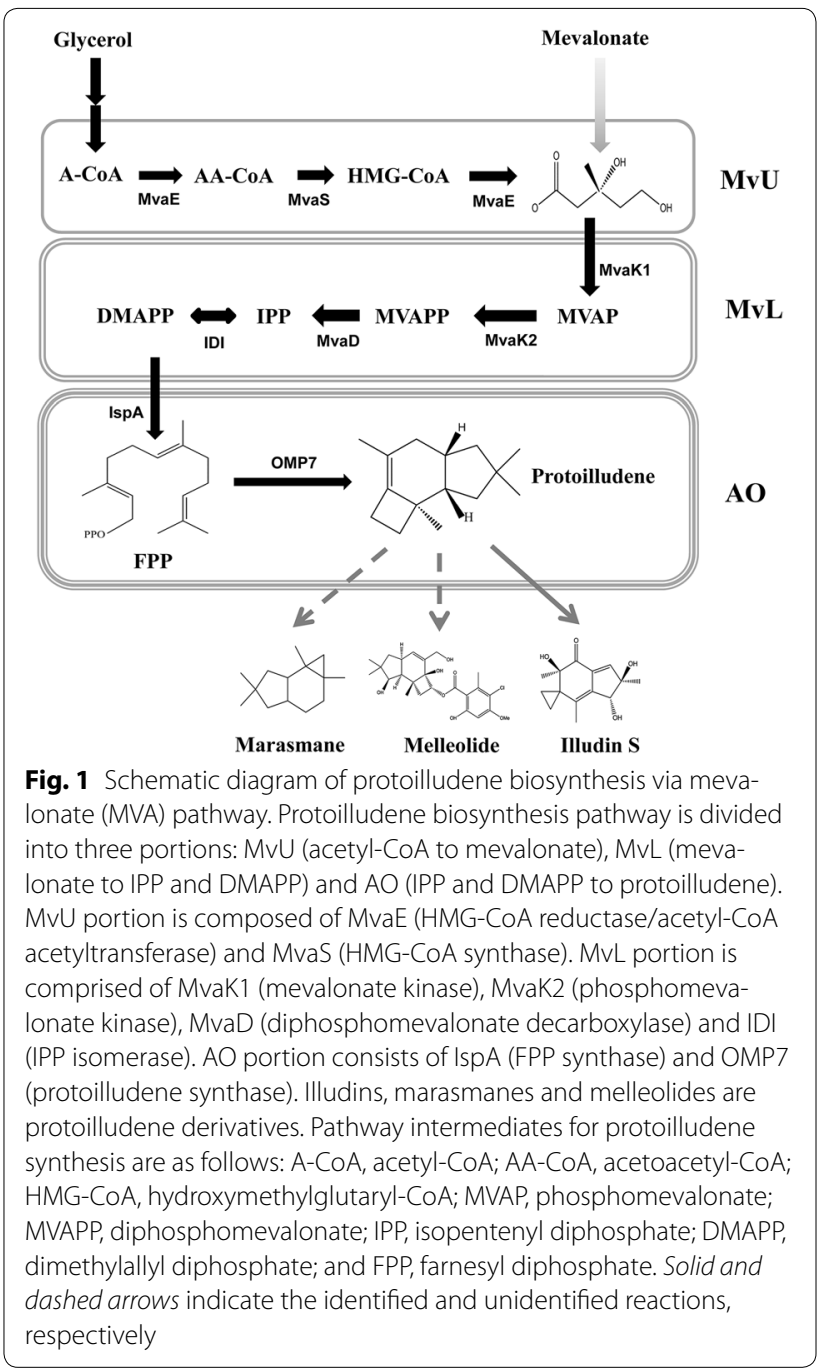

\section{Results and discussion}

Establishment of a protoilludene biosynthesis pathway in E. coli

Up to now, 6 protoilludene synthases from three species were identified (Additional file 1: Table S1) [7-9]. Among them, O. olearius protoilludene synthase (OMP7) exhibits the highest catalytic efficiency which is higher than its homologs OMP6 and Stehi1|73029 by 10 and 30 times, respectively. In order to synthesize protoilludene in $E$. coli, a codon-optimized $O M P 7$ gene was assembled with $E$. coli FPP synthase gene (ispA) to construct plasmid pTAO (Fig. 2a). It was transformed into E. coli DH5 $\alpha$, resulting in the strain $E$. coli $\mathrm{AO}$. This strain was then cultivated at $30{ }^{\circ} \mathrm{C}$ for $48 \mathrm{~h}$ in $2 \mathrm{YT}$ medium containing $2 \%$ (v/v) of glycerol with overlaying $1 \mathrm{~mL}$ decane. Gas chromatographic (GC) analysis showed a new peak which was identified as protoilludene by gas chromatograph-mass 
spectrometer (GC-MS), and corresponded to $1.14 \mathrm{mg} / \mathrm{L}$ of protoilludene. The tiny production could be ascribed to an insufficient supply of IPP and DMAPP from the native MEP pathway.

Thus, the protoilludene synthesis plasmid pTAO was co-transformed with plasmid pSNA [14], which encodes a hybrid exogenous MVA pathway for sufficient supply of IPP and DMAPP, into $E$. coli DH5 $\alpha$, resulting in the strain E. coli AO/NA. Gas chromatographic analysis showed a specific peak with retention time of $5.7 \mathrm{~min}$, which was subsequently confirmed as protoilludene by GCMS (Fig. 2b). For $48 \mathrm{~h}$ of culture, the strain E. coli AO/ NA produced $517 \mathrm{mg} / \mathrm{L}$ of protoilludene with an undesired accumulation of mevalonate as much as $571 \mathrm{mg} / \mathrm{L}$ (Fig. 2c), indicating the suboptimal performance of MVA pathway encoded by pSNA. It is thus required to redesign the MVA pathway, especially the lower portion of the MVA pathway for protoilludene production.

\section{Optimization of the MvL portion of the MVA pathway by sequential order permutation}

Expression levels of genes in an operon are known to be affected by their position within the operon [13]. If a gene is located at the tail end of the operon, its expression level is generally lower. Thus, relative expression levels of multi-genes in an operon can be affected by the sequential order of genes in the operon. A specific metabolic pathway encoded by an operon can be optimized by positional modulation of the constituent genes in the operon. Such an approach has been successfully applied to optimization of zeaxanthin synthetic pathway in Bacillus subtilis [15]. The MvL portion of pSNA is composed of four genes SnMvaK1, SnMvaK2 and SnMvaD from Streptococcus pneumoniae, and IDI from E. coli [10]. Optimization of the MvL portion was performed by sequential order permutation of three genes SnMvaK1, SnMvaK2 and $S n M v a D$. The four genes were assembled in a "Biobrick" [16] fashion to construct six sequential order permutated lower MVA pathway plasmids $\left(\mathrm{pSMvL}_{1-6}\right)$ based on pSTV28 vector (Fig. 3a). The strains E. coli $\mathrm{AO} / \mathrm{MvL}_{1-}$ ${ }_{6}$ resulting from the co-transformation of $\mathrm{pSMvL}_{1-6}$ and pTAO were evaluated for protoilludene production with supplementation of $4 \mathrm{mM}(592.6 \mathrm{mg} / \mathrm{L})(\mathrm{R}, \mathrm{S})$-mevalonate (Fig. 3b). The protoilludene production varied with the sequential order permutation in the MvLs. The highest protoilludene production of $137 \mathrm{mg} / \mathrm{L}$ was obtained from $E$. coli $\mathrm{AO} / \mathrm{MvL}_{2}$, whereas $E$. coli $\mathrm{AO} / \mathrm{MvL}_{4-6}$ produced low titers of protoilludene below $25 \mathrm{mg} / \mathrm{L}$. Around $80 \mathrm{mg} / \mathrm{L}$ of protoilludene was produced from $E$. coli $\mathrm{AO} /$ $\mathrm{MvL}_{1,3}$. Residual amounts of mevalonate in the culture broth were measured at the end of the culture to observe the consumption by the strains harboring these sequential order permutated plasmids (Additional file 1: Fig.

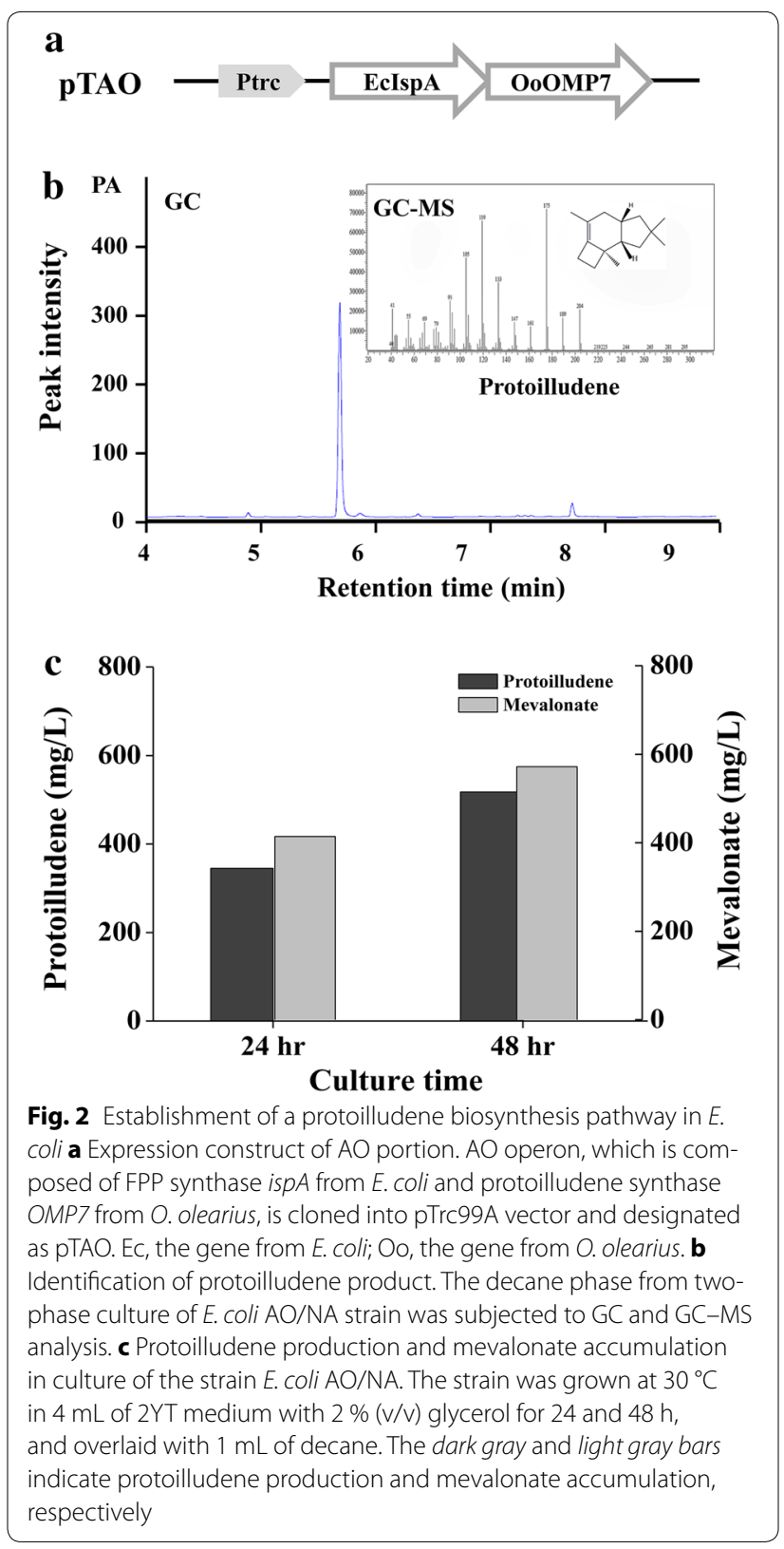

S2). As expected, the mevalonate consumption generally corresponded to the protoilludene production. The residual mevalonate concentrations in culture of $E$. coli $\mathrm{AO} / \mathrm{MvL}_{4-6}$ were as high as $3 \mathrm{mM}(438.5 \mathrm{mg} / \mathrm{L})$, whereas the concentrations in E. coli $\mathrm{AO} / \mathrm{MvL}_{1,3}$ and $\mathrm{AO} / \mathrm{MvL}_{2}$ were as low as around $1.7 \mathrm{mM}(248.5 \mathrm{mg} / \mathrm{L})$ and $1.3 \mathrm{mM}$ (190.0 mg/L), respectively. Therefore, the lower MVA pathway plasmid $\mathrm{pSMvL}_{2}$ is found to have an optimized gene order for the best performance of the MvL portion, and its order of SnMvaK1-SnMvaD-SnMvaK2-IDI is interestingly consistent with arrangement of the native genes in S. pneumoniae (GenBank: AE007317.1). 


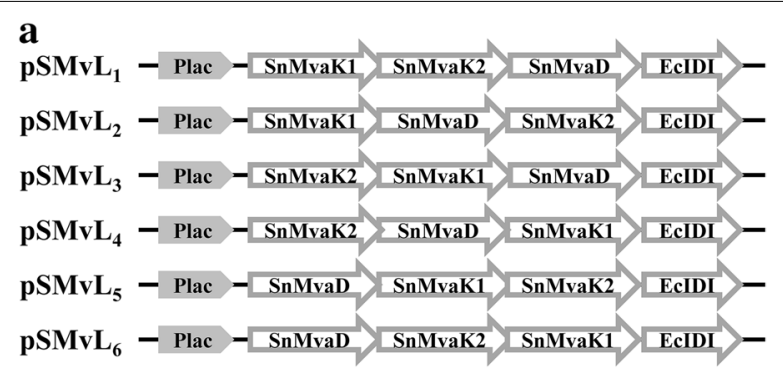

b

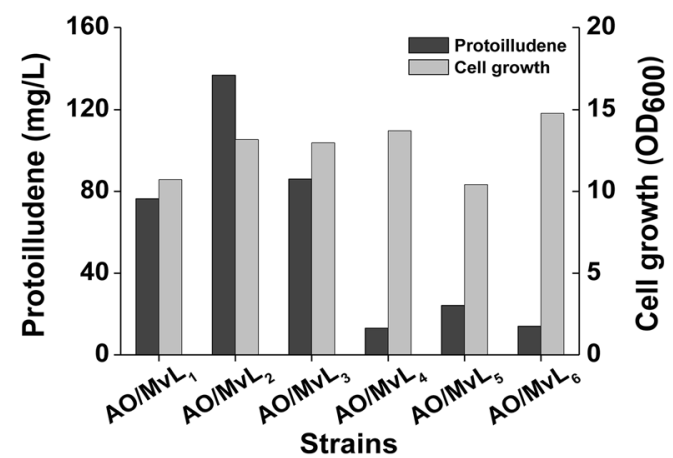

Fig. 3 Optimization of the lower (MvL) portion of MVA pathway by sequential order permutation. a Expression constructs of the sequential order permutated MvL portions. MvL operon, containing MvaK1, MvaK2 and MvaD from S. pneumoniae, and IDI from E. coli, is cloned into pSTV28 vector. Sn, the gene from S. pneumoniae; Ec, the gene from E. coli; Plac, lac promoter. $\mathbf{b}$ Effect of sequential order permutated MvLs on protoilludene production and cell growth. The strains were grown for $48 \mathrm{~h}$ at $30^{\circ} \mathrm{C}$ in $4 \mathrm{~mL}$ of $2 \mathrm{YT}$ medium containing $2 \%$ ( $\mathrm{v} / \mathrm{v})$ glycerol and $4 \mathrm{mM}$ mevalonate with overlay of $1 \mathrm{~mL}$ decane. The dark and light gray bars indicate protoilludene production and cell growth, respectively

\section{Coordination of MvU and MvL portions of MVA pathway for protoilludene production}

To optimize the synthesis of mevalonate, the MvU portion of the MVA pathway was cloned into three plasmids with different copy numbers and promoters, pBBR1MCS-2 (6-8 copies and lac promoter), pSTV28 (10-15 copies and lac promoter), and pTrc99A (20-30 copies and trc promoter) [12], which were designated as $\mathrm{pBMvU}_{\mathrm{L}}(\mathrm{LOW}), \mathrm{pSMvU}_{\mathrm{M}}(\mathrm{MEDIUM})$ and $\mathrm{pTMvU}_{\mathrm{H}}$ (HIGH), respectively (Fig. 4a). The alternations of copy number and promoter led to the differentiation of mevalonate producing capacity in a range of $104-215 \mathrm{mg} / \mathrm{L}$ per $\mathrm{OD}_{600}$ at $48 \mathrm{~h}$ (Fig. 4b), although there was no significant difference in cell growth among these three strains (Additional file 1: Fig. S3).

Both MvUs and MvLs were then expressed in all combinations in E. coli to find an optimal combination of the two portions for protoilludene production. As the $\mathrm{MvU}_{\mathrm{L}}$ plasmid $\left(\mathrm{pBMvU} \mathrm{L}_{\mathrm{L}}\right.$ ) is compatible with the lower MVA portion plasmids $\left(\mathrm{pSMvL}_{1-6}\right)$ and the protoilludene plasmid (pTAO), E. coli can be transformed with the three plasmids for the combination of $\mathrm{MvUs}$ and MvLs in protoilludene production. However, the $\mathrm{MvU}_{M}$ plasmid $\left(\mathrm{pSMvU}_{\mathrm{M}}\right)$ is not compatible with $\mathrm{pSMvL}_{1-6}$ derived from the same cloning vector (pSTV28) and $\mathrm{MvU}_{\mathrm{M}}$ and $\mathrm{MvL}_{1-6}$ are combined in $\mathrm{pSMvL}_{1-6}-\mathrm{MvU}_{\mathrm{M}}$ (Additional file 1: Fig. S4). The $\mathrm{MvU}_{\mathrm{H}}$ portion was cloned into pTAO plasmid, resulting in pTAO- $\mathrm{MvU}_{\mathrm{H}}$, because the $\mathrm{MvU}_{\mathrm{H}}$ plasmid $\left(\mathrm{pTMvU} \mathrm{H}_{\mathrm{H}}\right.$ ) is not compatible with the same vector originated pTAO plasmid (Additional file 1: Fig. S4). Escherichia coli $\mathrm{AO} / \mathrm{H} 1-\mathrm{H} 6$ strains harboring pTAO- $\mathrm{MvU}_{\mathrm{H}}$ and $\mathrm{pSMvL}_{1-6}$ produced a little amount of protoilludene ( $<35 \mathrm{mg} / \mathrm{L}$; Fig. 4c), accompanying with accumulation of a large amount of mevalonate ( $>1300 \mathrm{mg} / \mathrm{L}$ ). It indicated the $\mathrm{MvU}_{\mathrm{H}}$ produced too much mevalonate beyond the capacity of MvLs and the metabolic unbalance between $\mathrm{MvU}_{\mathrm{H}}$ and MvLs caused even a significant decrease of cell growth (Additional file 1: Table S2). In contrast, there was no significant accumulation of mevalonate in the strains of E. coli AO/L1-L6 (pTAO/pSMvL ${ }_{1-6} / \mathrm{pBMvU}_{\mathrm{L}}$ ) and $E$. coli $\mathrm{AO} / \mathrm{M} 1-\mathrm{M} 6$ $\left(\mathrm{pTAO} / \mathrm{pSMvL}_{1-6}-\mathrm{MvU}_{\mathrm{M}}\right)$, which suggested the lower capacity of the upper portions $\mathrm{MvU}_{\mathrm{L}}$ and $\mathrm{MvU}_{\mathrm{M}}$ than the lower portion MvLs (Fig. 4d). In contrast, strains E. coli AO/L1-L6 and E. coli AO/M1-M6 did not exhibit significant mevalonate accumulation (Fig. 4d). However, the poor mevalonate supply from $\mathrm{MvU}_{\mathrm{L}}$ compared to $\mathrm{MvU}_{\mathrm{M}}$ seems to restrict the protoilludene production. The highest protoilludene production of $721 \mathrm{mg} / \mathrm{L}$ was observed in $E$. coli $\mathrm{AO} / \mathrm{M} 2$, which represented a 1.4-fold increase to the production from $E$. coli $\mathrm{AO} / \mathrm{NA}$.

\section{Homolog substitution of the lower MVA portion genes}

Kinetic properties of homolog enzymes from different organisms are generally distinct from each other. Homolog enzymes of the lower MVA portion have also different kinetic properties. For example, S. pneumoniae mevalonate kinase (SnMvaK1) is subject to allosteric regulation by diphosphomevalonate, whereas $S$. aureus mevalonate kinase (SaMvaK1) without the allosteric regulation is competitively inhibited by isoprenyl diphosphates (DMAPP, IPP and FPP) [17, 18]. A metabolic pathway of interest can be improved by substituting a problematic constituent enzyme with its homolog with a desirable property [14]. In order to further improve the mevalonate pathway, the genes of the lower MVA portion $\mathrm{MvL}_{2}$ in $\mathrm{pSMvL}_{2}$ were substituted with their homologs from $S$. aureus, resulting into a new set of $\mathrm{pSMvL}_{7-13}$ plasmids (Fig. 5a). The effect of the lower MVA portions $\mathrm{MvL}_{7-13}$ on protoilludene production was investigated in combination with the upper MVA pathway portions $\mathrm{MvU}_{\mathrm{M}}$ and $\mathrm{MvU}_{\mathrm{H}}$ in the same manner used in Fig. 4c. The upper MVA portion $\mathrm{MvU}_{\mathrm{L}}$ was excluded in this study because it was suspected to produce insufficient amount 

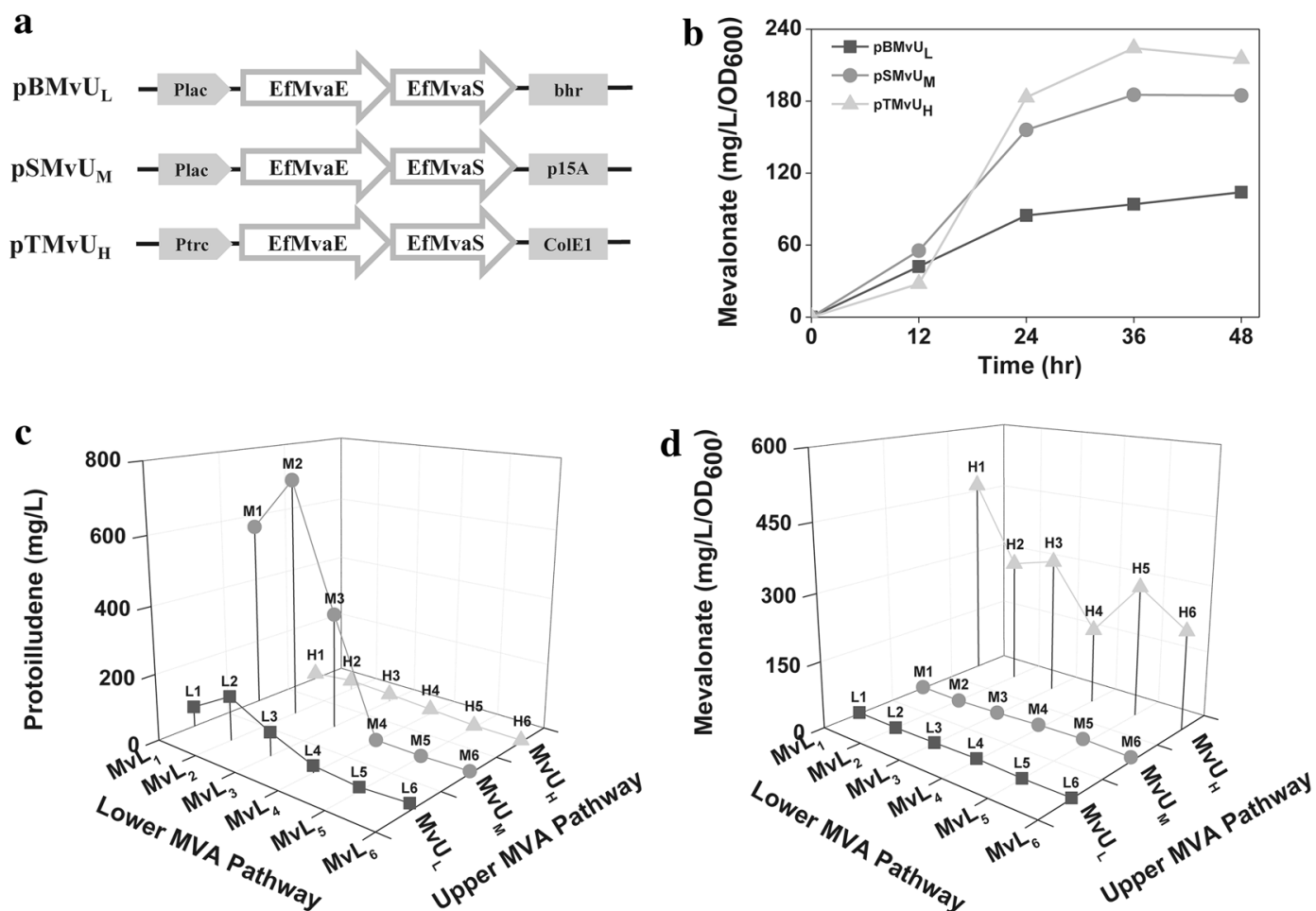

Fig. 4 Coordination of the lower (MvL) and upper (MvU) portions of MVA pathway for protoilludene production. a Expression constructs of the $\mathrm{MvU}$ portions with alternations of promoter and copy-number. MvU operon consists of MvaE and MvaS from E. faecalis. The two genes are cloned into pBBR1MCS-2, pSTV28 and pTrc99A vectors, which are designated as $\mathrm{pBMVU}_{L^{\prime}} \mathrm{pSMvU}_{\mathrm{M}}$ and $\mathrm{pTMv}_{\mathrm{H}^{\prime}}$, respectively. Ef, the gene from E. faecalis; Plac and Ptrc, lac promoter and trc promoter, respectively. Rectangles show replication origin of each plasmid. $\mathbf{b}$ Mevalonate production capacity of recombinant $E$. coli harboring each of $\mathrm{pBMvU}_{L}, \mathrm{pSMv}_{\mathrm{M}}$ and $\mathrm{pTMv}_{H}$. The strains were cultured in $2 \mathrm{YT}$ medium at $30^{\circ} \mathrm{C}$ for $48 \mathrm{~h}$. $\mathbf{c}$ and $\mathbf{d}$ Effect of combinations of $\mathrm{MvU}_{\mathrm{L}, \mathrm{M}, \mathrm{H}}$ and $\mathrm{MvL}_{1-6}$ on protoilludene production and mevalonate accumulation. The culture were carried out in $4 \mathrm{~mL}$ of $2 \mathrm{YT}$ medium containing $2 \%(\mathrm{v} / \mathrm{v})$ glycerol with overlay of $1 \mathrm{~mL}$ decane at $30^{\circ} \mathrm{C}$ for $48 \mathrm{~h}$

of mevalonate for high production of protoilludene. The plasmids $\mathrm{pSMvL}_{7-13}-\mathrm{MvU}_{\mathrm{M}}$ were constructed to combine the upper portion $\mathrm{MvU}_{\mathrm{M}}$ and the lower portions $\mathrm{MvL}_{7-}$ ${ }_{13}$ (Additional file 1: Fig. S4). The combinations of $\mathrm{MvU}_{\mathrm{H}}$ and $\mathrm{MvL}_{7-13}$ were conducted by co-transformation of pTAO- $\mathrm{MvU}_{\mathrm{H}}$ and $\mathrm{pSMvL}_{7-13}$. Interestingly, the strains E. coli $\mathrm{AO} / \mathrm{M} 7\left(\mathrm{pTAO} / \mathrm{pSMvL}_{7}-\mathrm{MvU}_{\mathrm{M}}\right.$ ) and E. coli $\mathrm{AO} /$ $\mathrm{H} 7$ (pTAO- $\mathrm{MvU}_{\mathrm{H}}$ and $\mathrm{pSMvL}_{7}$ ), containing the lower MVA portion $\mathrm{MvL}_{7}$ with homolog substitution of $\mathrm{SaM}$ $v a K 1$ only, produced the enhanced protoilludene production of 1199 and $740 \mathrm{mg} / \mathrm{L}$, respectively (Fig. 5b and Additional file 1: Table S3). Other homolog substitutions failed to improve production of protoilludene (Fig. 5b). As the homolog substitution of SaMvaK1 with no allosteric inhibition by diphosphomevalonate is effective for protoilludene production, it is suspected the accumulation of diphosphomevalonate in the strain E. coli $\mathrm{AO} /$ M2 harboring the lower MVA portion $\mathrm{MvL}_{2}$ due to some bottleneck in the conversion of diphosphomevalonate to IPP by diphosphomevalonate decarboxylase.

\section{Conclusions}

It is demonstrated the feasibility of producing protoilludene in engineered E. coli. Heterologous expression of the MVA pathway encoded by pSNA enabled the strain E. coli AO/NA to produce $517 \mathrm{mg} / \mathrm{L}$ of protoilludene, but mevalonate was accumulated in a significant amount as $571 \mathrm{mg} / \mathrm{L}$ due to the unbalanced upper and lower portions of the MVA pathway. To create a balanced efficient MVA pathway, we sequentially permuted the order of genes in the lower portion of the MVA pathway (MvL) and coordinated their expression with the upper portion of the MVA pathway (MvU) by alternations of copy-number and promoter of plasmids. Through this approach, $721 \mathrm{mg} / \mathrm{L}$ of protoilludene was produced with reduced accumulation of mevalonate in the strain E. coli AO/M2. The substitution of mevalonate kinase from $S$. pneumoniae with the homolog from $S$. aureus further increased protoilludene production to $1199 \mathrm{mg} / \mathrm{L}$. These results suggest that the optimized MVA pathway is efficient to supply IPP and 

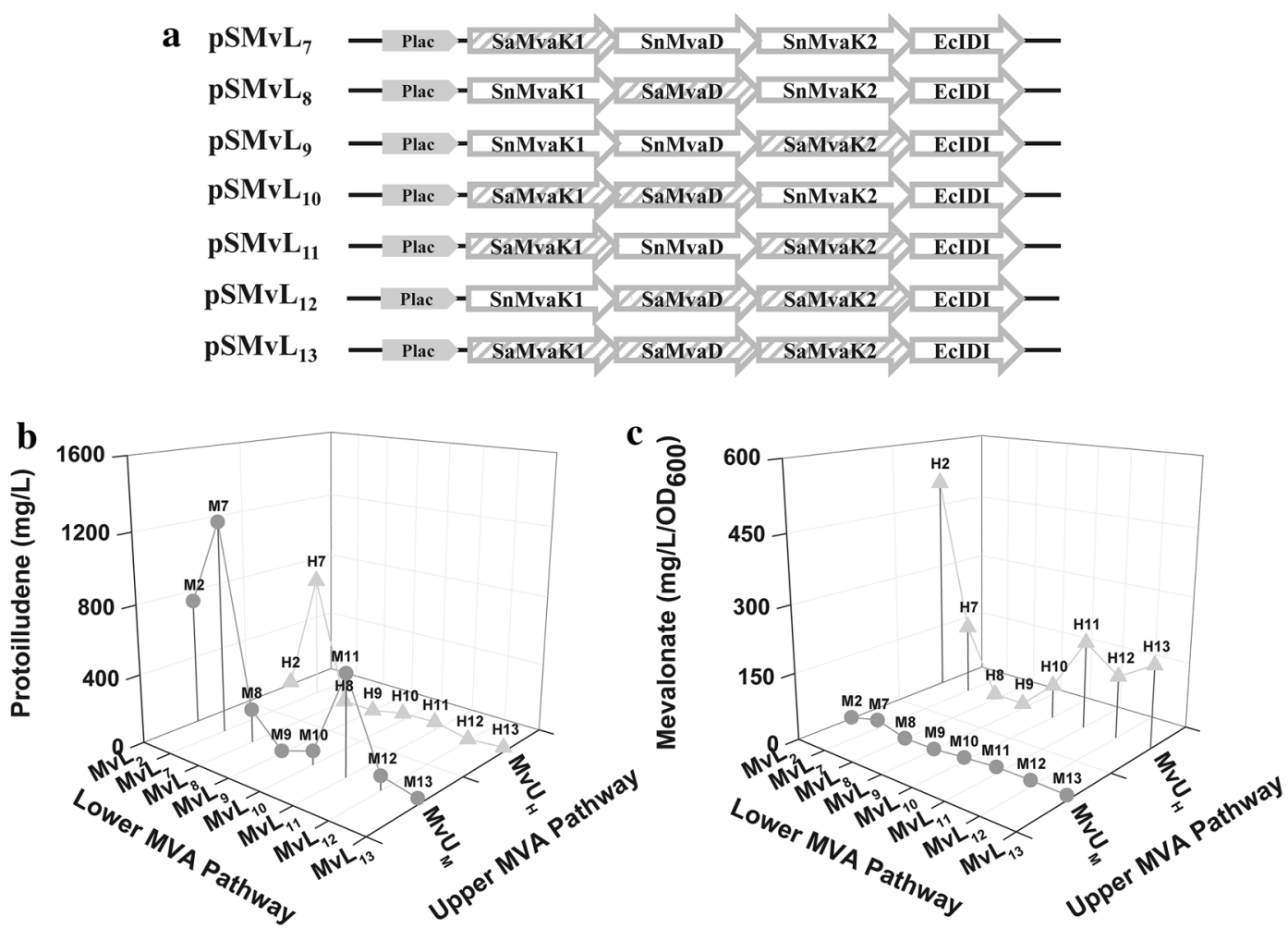

Fig. 5 Optimization of MVA pathway by homolog substitution for the lower portion genes. a Expression constructs of the homolog substituted MvL portions. The homolog genes are from S. aureus and represented with prefixion of "Sa" to the gene name. $\mathbf{b}$ and $\mathbf{c}$ Effect of combinations of $\mathrm{MvU}_{\mathrm{M}, \mathrm{H}}$ and $\mathrm{MvL}_{7-13}$ on protoilludene production and mevalonate accumulation. The culture were carried out in $4 \mathrm{~mL}$ of $2 \mathrm{YT}$ medium containing $2 \%(\mathrm{v} / \mathrm{v})$ glycerol with overlay of $1 \mathrm{~mL}$ decane at $30^{\circ} \mathrm{C}$ for $48 \mathrm{~h}$

DMAPP for protoilludene production and also can serve as a platform IPP/DMAPP synthesis pathway for production of other valuable terpenes.

\section{Methods}

\section{Bacterial strains and growth conditions}

Escherichia coli DH5 $\alpha$ were grown in $2 \mathrm{YT}$ medium (16 $\mathrm{g}$ tryptone, $10 \mathrm{~g}$ yeast extract, and $5 \mathrm{~g}$ sodium chloride per $1 \mathrm{~L}$ ) at $37{ }^{\circ} \mathrm{C}$ for plasmid construction, and at $30{ }^{\circ} \mathrm{C}$ for protoilludene production. The seed culture grown overnight at $37{ }^{\circ} \mathrm{C}$ was inoculated with an optical density at $600 \mathrm{~nm}\left(\mathrm{OD}_{600}\right)$ of 0.1 into $2 \mathrm{YT}$ medium containing $2 \%(\mathrm{v} / \mathrm{v})$ glycerol. Escherichia coli strains (Table 1) harboring the lower portion of the MVA pathway were cultured with addition of $4 \mathrm{mM}$ mevalonate. Ampicillin $(100 \mu \mathrm{g} / \mathrm{mL})$, chloramphenicol $(50 \mu \mathrm{g} / \mathrm{mL})$, kanamycin $(50 \mu \mathrm{g} / \mathrm{mL})$ and $0.2 \mathrm{mM}$ IPTG were added as required. To harvest protoilludene produced during culture, $1 \mathrm{~mL}$ of decane was initially overlaid on $4 \mathrm{~mL}$ of culture broth. Cell growth was determined by measuring the $\mathrm{OD}_{600}$. All experiments were carried out in duplicate.
Table 1 Strains used in this study

\begin{tabular}{|c|c|c|}
\hline Names & Descriptions & Sources \\
\hline E. coli AO & E. coli DH5a harboring pTAO & This study \\
\hline E. coli AO/NA & E. coli DH5a harboring pTAO and pSNA & This study \\
\hline E. coli $\mathrm{AO} / \mathrm{MvL}_{1-6}$ & $\begin{array}{l}\text { E. coli DH5a harboring pTAO and } \\
\text { pSMvL }_{1-6}\end{array}$ & This study \\
\hline E. coli AO/L1-L6 & $\begin{array}{l}\text { E. coli DH5a harboring } \mathrm{pTAO}, \mathrm{pSMvL}_{1-6} \\
\text { and } \mathrm{pBMvU}_{\mathrm{L}}\end{array}$ & This study \\
\hline E. coli AO/M1-M13 & $\begin{array}{l}\text { E. coli DH5a harboring pTAO and } \\
\mathrm{pSMVL}_{1-13}-\mathrm{MvU}_{M}\end{array}$ & This study \\
\hline E. coli AO/H1-H13 & $\begin{array}{l}\text { E. coli DH5a harboring } \mathrm{pTAOMvU}_{H} \text { and } \\
\mathrm{pSMvL}_{1-13}\end{array}$ & This study \\
\hline
\end{tabular}

This table is a brief description of strains used in this study. For more detailed information, refer to Additional file 1: Table S4

\section{Construction of plasmids}

Basic molecular biology procedures, including restriction enzyme digestion and bacterial transformation, were carried out as described in the literature [19]. DNA fragments were amplified by PCR using Pfu DNA polymerase (SolGent, Daejeon, Korea) according to the manufacturer's instructions. BglBricks assembly [16] was applied for 
construction of various plasmids. The schematic diagram of the constructs is shown in figures and the detailed construction process was depicted in Additional file 1. All plasmids and primers used in this study are described in Additional file 1: Table S4.

\section{Identification and quantification of protoilludene}

The decane phase of the two-phase culture was collected and centrifuged for $10 \mathrm{~min}$ at 12,000 rpm to remove cell debris, and subsequently subjected to gas chromatography (GC) and gas chromatography-mass spectrometry (GC-MS). The production of protoilludene was quantified using an Agilent Technologies 7890A gas chromatograph equipped with a flame ionization detector (FID). One $\mu \mathrm{L}$ of sample was injected at a split ratio of 1:10, and separated on a 19091 N-133 HP-INNOWAX column (length, $30 \mathrm{~m}$; internal diameter, $0.25 \mathrm{~mm}$; film thickness, $250 \mu \mathrm{m})$. The oven temperature was initially held at $80{ }^{\circ} \mathrm{C}$ for $1 \mathrm{~min}$ and was increased at a rate of $10^{\circ} \mathrm{C} / \mathrm{min}$ to $250^{\circ} \mathrm{C}$, where it was held for $1 \mathrm{~min}$. Nitrogen was used as the carrier gas with an inlet pressure of $39 \mathrm{psi}$. The detector temperature was maintained at $260{ }^{\circ} \mathrm{C}$. GC-MS analysis was run on a GCMS-2010 ultra mass spectrometer (Shimadzu, Tokyo, Japan). Purified protoilludene was used as the standard compound to construct the standard curve $\left(R^{2}>0.99\right)$ for the estimation of protoilludene production (Additional file 1: Fig. S1).

\section{Quantification of mevalonate}

Mevalonate concentration was determined by GC analysis. Culture filtrate was adjusted to $\mathrm{pH} 2$ with $3 \mathrm{M} \mathrm{HCl}$, incubated at $45{ }^{\circ} \mathrm{C}$ for $1 \mathrm{~h}$, saturated with anhydrous $\mathrm{Na}_{2} \mathrm{SO}_{4}$, and extracted with ethyl acetate. The resulting samples were analyzed for mevalonate concentration using an Agilent Technologies 7890A gas chromatograph. The analytical temperature of the GC was controlled at an initial temperature of $180{ }^{\circ} \mathrm{C}$ for $1 \mathrm{~min}$, then ramped to $200{ }^{\circ} \mathrm{C}$ gradually at $2.5^{\circ} \mathrm{C} / \mathrm{min}$ and held for $2 \mathrm{~min}$. The detector temperature was maintained at $260^{\circ} \mathrm{C}$.

\section{Additional file}

Additional file 1: Construction of plasmids. Table S1. Comparison of protoilludene synthases reported in literatures. Table S2. Cell growth of recombinant $E$. coli harboring MVA pathway engineered in a way of various combinations of $\mathrm{MvU}_{\mathrm{L}, \mathrm{M}, \mathrm{H}}$ and $\mathrm{MvL}_{1-6}$. Table S3. Cell growth of recombinant $E$. coli harboring MVA pathway engineered with combinations of $\mathrm{MvU}_{\mathrm{MH}}$ and $\mathrm{MVL}_{217-13}$. Table S4. Strains, plasmids and primers used in this study. Figure S1. GC-FID standard curve of protoilludene. Figure S2. Residual mevalonate in culture of the strains E. coli AO/MvL with exogenous addition of mevalonate. Figure S3. Cell growth of $E$. coli strains harboring $\mathrm{pBMv}_{L^{\prime}} \mathrm{pSMv}_{\mathrm{M}}$ and $\mathrm{pTMv} \mathrm{U}_{H}$. Figure S4. Schematic diagram of $\mathrm{PSMvL}_{1-13}-\mathrm{MvU}_{\mathrm{M}}$ and $\mathrm{pTAOMvU_{H }}$.

\section{Authors' contributions}

SWK and CW conceived the idea and designed the experiments. LY carried out the experiments. LY, CW and JZ analyzed the data. LY, SWK and CW drafted the manuscript. All authors read and approved the final manuscript.

\section{Author details}

1 Division of Applied Life Science (BK21 Plus Program), PMBBRC, Gyeongsang National University, Jinju 660-701, Korea. ${ }^{2}$ Faculty of Life Science and Food Engineering, Huaiyin Institute of Technology, Huai'an 223003, The People's Republic of China.

\section{Acknowledgements}

This work was supported by a Grant (NRF-2013R1A1A2008289) and a Grant (NRF-2012M1A2A2671831) from the National Research Foundation, MSIP and a Grant from the Next-Generation BioGreen 21 Program (SSAC, Grant\#: PJ01106201), Rural Development Administration, Korea.

\section{Competing interests}

The authors declare that they have no competing interests.

Received: 10 November 2015 Accepted: 4 January 2016

Published online: 19 January 2016

\section{References}

1. Reina M, Orihuela JC, Gonzalez-Coloma A, de Ines C, de la Cruz M, del Val GA, Torno JR, Fraga BM. Four illudane sesquiterpenes from coprinopsis episcopalis. Phytochemistry. 2004;65:381-5.

2. Wang XN, Shen JH, Du JC, Liu JK. Marasmane sesquiterpenes isolated from Russula foetens. J Antibiot. 2006;59:669-72 (Tokyo).

3. Bohnert M, Nutzmann HW, Schroeckh V, Horn F, Dahse HM, Brakhage AA, Hoffmeister D. Cytotoxic and antifungal activities of melleolide antibiotics follow dissimilar structure-activity relationships. Phytochemistry. 2014;105:101-8.

4. Schobert R, Knauer S, Seibt S, Biersack B. Anticancer active illudins: recent developments of a potent alkylating compound class. Curr Med Chem. 2011;18:790-807.

5. Wawrzyn GT. Discovery and characterization of sesquiterpenoid biosynthetic pathways from Basidiomycota. Retrieved from the University of Minnesota Digital Conservancy. http://hdl.handle.net/11299/162701.

6. Martin VJ, Pitera DJ, Withers ST, Newman JD, Keasling JD. Engineering a mevalonate pathway in Escherichia coli for production of terpenoids. Nat Biotechnol. 2003;21:796-802.

7. Engels B, Heinig U, Grothe T, Stadler M, Jennewein S. Cloning and characterization of an Armillaria gallica cDNA encoding protoilludene synthase, which catalyzes the first committed step in the synthesis of antimicrobial melleolides. J Biol Chem. 2011;286:6871-8.

8. Wawrzyn GT, Quin MB, Choudhary S, Lopez-Gallego F, Schmidt-Dannert C. Draft genome of Omphalotus olearius provides a predictive framework for sesquiterpenoid natural product biosynthesis in basidiomycota. Chem Biol. 2012;19:772-83.

9. Quin MB, Flynn CM, Wawrzyn GT, Choudhary S, Schmidt-Dannert C. Mushroom hunting by using bioinformatics: application of a predictive framework facilitates the selective identification of sesquiterpene synthases in basidiomycota. Chembiochem. 2013;14:2480-91.

10. Nowroozi FF, Baidoo EE, Ermakov S, Redding-Johanson AM, Batth TS, Petzold CJ, Keasling JD. Metabolic pathway optimization using ribosome binding site variants and combinatorial gene assembly. Appl Microbiol Biotechnol. 2014;98:1567-81.

11. Wang C, Yoon SH, Jang HJ, Chung YR, Kim JY, Choi ES, Kim SW. Metabolic engineering of Escherichia coli for alpha-farnesene production. Metab Eng. 2011;13:648-55.

12. Anthony JR, Anthony LC, Nowroozi F, Kwon G, Newman JD, Keasling JD. Optimization of the mevalonate-based isoprenoid biosynthetic pathway in Escherichia coli for production of the anti-malarial drug precursor amorpha-4,11-diene. Metab Eng. 2009;11:13-9. 
13. Lim HN, Lee $Y$, Hussein R. Fundamental relationship between operon organization and gene expression. Proc Natl Acad Sci USA 2011;108:10626-31.

14. Yoon SH, Lee SH, Das A, Ryu HK, Jang HJ, Kim JY, Oh DK, Keasling JD, Kim SW. Combinatorial expression of bacterial whole mevalonate pathway for the production of beta-carotene in E. coli. J Biotechnol. 2009;140:218-26.

15. Nishizaki T, Tsuge K, Itaya M, Doi N, Yanagawa H. Metabolic engineering of carotenoid biosynthesis in Escherichia coli by ordered gene assembly in Bacillus subtilis. Appl Environ Microbiol. 2007;73:1355-61.

16. Anderson JC, Dueber JE, Leguia M, Wu GC, Goler JA, Arkin AP, Keasling JD. BglBricks: a flexible standard for biological part assembly. J Biol Eng 2010;4:1.
17. Andreassi JL 2nd, Dabovic K, Leyh TS. Streptococcus pneumoniae isoprenoid biosynthesis is downregulated by diphosphomevalonate: an antimicrobial target. Biochemistry. 2004;43:16461-6.

18. Voynova NE, Rios SE, Miziorko HM. Staphylococcus aureus mevalonate kinase: isolation and characterization of an enzyme of the isoprenoid biosynthetic pathway. J Bacteriol. 2004;186:61-7.

19. Sambrook J, Russell DW. Molecular cloning: a laboratory manual. New York: Cold Spring Harbor Laboratory Press; 2001.
Submit your next manuscript to BioMed Central and we will help you at every step:

- We accept pre-submission inquiries

- Our selector tool helps you to find the most relevant journal

- We provide round the clock customer support

- Convenient online submission

- Thorough peer review

- Inclusion in PubMed and all major indexing services

- Maximum visibility for your research

Submit your manuscript at www.biomedcentral.com/submit
(O) BioMed Central 